\title{
Dietary lipids and the brain during development and ageing
}

\author{
Ricardo Uauy ${ }^{1,2}$ and Alan Dangour ${ }^{2}$
}

'Public Health Nutrition Division, Instituto Nutrición y Tecnología de Alimentos (INTA), Universidad de Chile, Santiago,

Chile; ${ }^{2}$ Nutrition and Public Health Intervention Research Unit, London School of Hygiene \& Tropical Medicine,

London, UK

Abstract

The brain and retina are rich in long-chain polyunsaturated fatty acids (LCPs). Docosahexaenoic acid (C22:6 n-3; DHA) particularly has been shown to affect retinal and brain development in humans. Provision of n-3 LCPs in preterm and term babies is associated with enhanced retinal electrical responses to light stimuli and to a pattern of brain cortex-related visual acuity maturation that is similar to that observed in infants fed on human milk. Evidence supporting the potential importance of n-3 LCP consumption for good cognitive health in older age is also beginning to emerge. Cross-sectional and prospective data indicate that higher fatty fish and n-3 LCP consumption is associated with reduced risk of impaired cognitive function or dementia. The evidence suggests that n-3 LCPs act by inhibiting hepatic triglyceride synthesis as well as modulating eicosanoid function, inducing vascular relaxation, and diminishing inflammatory processes and platelet aggregation. This article reviews the role of LCPs in the brain during development and ageing, and the mechanisms that may explain the observed effects.

Keywords: n-3 fatty acids; docosahexaenoic acid; brain; retina; development; ageing

Abbreviations: AA: arachidonic acid; DHA: docosahexaenoic acid; DPA: docosapentaenoic acid; EPA: eicosapentaenoic acid; LA: linoleic acid; LCP: long-chain polyunsaturated fatty acid; LNA: $\alpha$-linolenic acid; PPAR: peroxisomal proliferator activated receptor; PS: phosphatidyl serine; PUFA: polyunsaturated fatty acid.

\section{Introduction}

$\mathrm{I}$ $\mathrm{t}$ is now clear that $\mathrm{n}-3$ long-chain polyunsaturated fatty acids (LCPs) are crucial for the development of the brain and retina in humans. Provision of $n-3$ LCPs in preterm and term babies is associated with enhanced retinal electrical responses to light stimuli, and to a pattern of brain cortexrelated visual acuity maturation that is similar to that observed in infants fed on human milk (1). Some results in young children suggest that neurodevelopment and cognitive abilities are also enhanced by early provision of n-3 LCPs (2). The mechanisms of action of n-3 LCPs are complex, but are slowly beginning to be understood. This paper examines the mechanisms for the potential effects of n-3 LCPs on brain development and ageing; these can be broadly grouped into three categories: membrane effects, modulation of eicosanoid production, and action on neurotropic factors and apoptosis. This paper also examines how these mechanisms could potentially operate during development and ageing.

\section{Membrane effects}

One of the most significant membrane effects of docosahexaenoic acid (DHA) is its role in the photoreceptor signal transduction process. In 1996, Litman and Mitchell reported that LCPs present in membrane phospholipid molecular species have significant effects on rhodopsin activation and related structural modifications (3). Rhodopsin, a membrane protein present in rod outer segment disc membranes, accounts for $90 \%$ of the protein content and functions as a photon receptor coupled to a $\mathrm{G}$ protein. The light-induced conformational change in rhodopsin triggers a biochemical cascade, finally leading to an increase in phosphodiesterase activity and a decrease in cyclic guanosine monophosphate (cGMP), which closes $\mathrm{Na}^{+}$channels in the photoreceptor disc membrane. The result is a hyperpolarization, increasing the negative charge of the plasma membrane, which is followed by a depolarization.

Membrane fatty acid composition affects the ability of photons to transform rhodopsin to the 
activated state. Activation of rhodopsin in response to light involves a transformation from metarhodopsin I to metarhodopsin II. The equilibrium constant of the di-DHA-acylated phosphatidylcholine is almost identical to that of native rod discs and is six times higher than that of di-myristic (saturated 14:0)-acylated phosphatidylcholine. This effect is mostly explained by the increase in membrane free volume, which results in greater mobility of rhodopsin within the lipid microenvironment. This most probably explains the change in G-protein activation and the corresponding enhanced signal transduction to photon stimuli (3). This corresponds with the physiological phenomenon of increased retinal sensitivity to light that is associated with enhanced DHA supply in the diet.

\section{Modulation of eicosanoid production}

A second mechanism by which LCP supplementation may affect different physiological functions is via their role in the production of various eicosanoids. Phospholipases liberate arachidonic acid (20:4 n-6; AA) and eicosapentaenoic acid (20:5 n-3; EPA) from membrane lipids, and through the action of cyclooxygenase or lipoxygenase, eicosanoid products are formed. Prostaglandins, prostacyclins, thromboxanes and leukotrienes derived from LCPs play a key role in modulating inflammation, cytokine release, immune response, platelet aggregation, vascular reactivity, thrombosis and allergic phenomena. The balance between AA and EPA in biological membranes is partly determined by dietary supply, and the $n-6: n-3$ ratio in phospholipids modulates the balance between prostanoids of the AA-derived 2 series and the EPAderived 3 series. Eicosanoids of the 2 series promote inflammation and platelet aggregation, and activate the immune response. In contrast, series-3 prostanoids tend to ameliorate these effects. This topic has been the subject of an excellent recent review (4).

\section{Neurotrophic factors and apoptosis}

DHA has definite effects on various neurotrophic factors. Cells cannot survive in isolation; neighbouring cells contribute to cellular and tissue-specific maturation by providing autocrine and paracrine growth factors. These polypeptide hormones, also called trophic factors, act in target cells by stimulating cell membrane-specific receptors that activate tyrosine kinase-mediated responses, which affect various biological functions such as cell survival, proliferation, growth arrest, differentiation, proliferation and programmed cell death (apoptosis). Cells in culture require some foetal calf or bovine serum in the media, and the absence of growth factors, typically present in serum, induces cell death by apoptosis. Cellular apoptosis is a wellorchestrated process in both the timing and sequence of various events, and apoptosis is crucial for tissue and organ health since it maintains the balance between cell growth and cell death necessary during development.

During embryonic and foetal life, the nervous system is shaped by multiple cell types, some of which suffer apoptotic death. For example, after birth, axonic projections from the right and left lateral geniculate nuclei are connected to neurons in the right and left occipital cortex in a complex manner, determining the structural basis for binocular vision. Cortical cells that fail to establish synapses with the axonic projections that provide sensory input from a specific hemi-retina (medial or lateral half) are discarded undergoing apoptosis (5).

Apoptosis is determined by the orchestrated expression of different genes. These genes can be grouped in two major categories: the regulators, which are a large family of structurally related proteins belonging to Bcl-2 family, and the executioners, also known as caspases. The latter group includes aspartic proteases involved in final degradation of key proteins, ultimately causing cell demise. Recent evidence indicates that DHA can inhibit or ameliorate cellular apoptosis of neural cells. For example, newborn rat retinal cells cultured in medium containing DHA survive and differentiate into photoreceptors, while those cultured in the absence of DHA have limited photoreceptor outer segment development and the cells eventually degenerate and die by apoptosis (6). Supplementation with DHA, but not AA or other fatty acids, prevents photoreceptor degeneration and death. The developmental timing for this phenomenon coincides with the histological evidence of active uptake and esterification of DHA into rod outer segment discs (7). An antiapoptotic effect of DHA has also been reported in a model of HL-60 cells with sphingosine as an inductor of apoptosis. This was also related to the uptake and esterification of DHA in phospholipids (8). The antiapoptotic effect apparently requires DHA incorporation into phospholipids, but the exact mechanism by which it occurs is still unclear. Kim et al. demonstrated that 
DHA can inhibit the apoptosis induced by serum starvation in 2A neurons (9). In this case, the antiapoptotic effect of DHA was correlated specifically with the accumulation of DHA in phosphatidyl serine (PS). In fact, the effect was blocked by growing cells in serine-free medium, which limits PS accumulation, suggesting that PS has a key role in the antiapoptotic effect of DHA.

Regulation of gene expression by LCPs occurs at the transcriptional level, and is mediated by transcription factors that bind cis-regulatory elements found in target genes. These transcription factors, which are activated by fatty acids, belong to the superfamily of nuclear receptors that mediate the action of vitamin $\mathrm{D}$, steroid and thyroid hormones. Two types of transcription factor responsive to polyunsaturated fatty acids (PUFAs) have been characterized; these are the peroxisomal proliferator activated receptor (PPAR) and the hepatic nuclear factor- $4 \alpha$. Recent studies have identified a number of protein coactivators that interact with nuclear receptors playing a role in the regulation of transcriptional activity. The formation of the binding site for the coactivator in the nuclear receptor is ligand dependent.

\section{Effects during brain development}

A large body of evidence now supports the proposal that the essential fatty acids, particularly the n-3 LCPs, are crucial for brain development in humans. Indeed, the high concentrations of n-3 LCPs, such as DHA in the cerebral cortex and the retina, suggest an important role for these fats: the dry weight of the human brain is predominantly lipid, with $22 \%$ of the cerebral cortex and $24 \%$ of white matter consisting of phospholipids. While the nature and concentration of proteins in the brain are determined by the genetic code, the fatty acid composition of brain phospholipids can be modified by diet $(10,11)$, highlighting the importance of adequate diet throughout the life-course to ensure optimal brain phospholipid composition.

To examine the conditional essentiality of $n-3$ LCPs, single- and multicompartmental kinetic analysis was used to assess fatty acid metabolism based on the flux of isotopes through the various plasma compartments as an indirect assessment of liver long-chain fatty acid formation. This has allowed determination of the relative quantitative contributions of linoleic acid (C18:2 n-6; LA) and $\alpha$-linolenic acid (C18:3 n-3; LNA) to the synthesis of desaturated and elongated long-chain fatty acids in the plasma of preterm and full-term infants soon after birth. In term infants LA plasma concentrations appeared to be near equilibrium, as a utilization of $2 \mathrm{~g}$ per day (predicted from the model) was similar to predicted LA intake $(2.1 \pm 0.8 \mathrm{~g}$ per day). The predicted LA need was in good agreement with an estimated mean of $2.3 \mathrm{~g}$ of LA derived from formula/breast milk intake data. These data indicate that the usual dietary provision of LA from formula or breast milk is sufficient to support tissue needs. LCP formation of in utero growth-restricted infants has also been explored, and a limited capacity for microsomal $\beta$-oxidation was identified in such infants; these infants accumulate docosapentaenoic acid (22:5 n-3 DPA) owing to impaired function of peroxisomes (12).

\section{Effects during brain ageing}

Levels of DHA in the ageing brain decrease (13) and these age-related changes appear to be associated with changes in nervous system function. For example, rats fed on a low-DHA diet for one or more generations show clear deficits in cognitive function (14). Evidence supporting the importance of adequate n-3 LCP consumption for good cognitive function in later life in humans is growing. Cross-sectional data have demonstrated that higher fish and n-3 LCP consumption is associated lower risks of cognitive impairment (15), while prospective data has shown that older people who consume more fish are less likely to suffer dementia $(16,17)$. The mechanisms that can explain these effects have not been fully elucidated. The actions of EPA in the inhibition of hepatic triglyceride synthesis, and the modulation of eicosanoid function to induce vascular relaxation, diminished inflammatory process and decreased platelet aggregation are likely to be crucial (18). These actions may, for example, be important in decreasing brain microinfarcts that are a cause of vascular cognitive impairment shown to be directly associated with cognitive decline $(19,20)$.

A further clue regarding the neuroprotective mechanisms of action of n-3 LCPs during ageing was recently demonstrated in a mouse model of ischaemic stroke which investigated the effect of DHA on two of the major causes of post-stroke neuronal injury, namely lipid peroxidation and leukocyte infiltration. These two actions were potently inhibited by a novel bioactive docosanoid derived from DHA (10-17S-docosatriene, 
Table I. Effect of docosahexaenoic acid (DHA) supplementation on the fatty acid composition of human foetal retinal explants after 14 days of incubation in bovine serum with $20 \mu \mathrm{M}$ DHA or none added

\begin{tabular}{lccc}
\hline \multirow{2}{*}{ Fatty acid } & \multicolumn{2}{c}{ DHA supplementation } & \\
\cline { 2 - 3 } & + & \multicolumn{2}{c}{ Ratio } \\
\cline { 2 - 3 } & $(\%)$ & $(\%)$ & \\
\hline Saturated & 52.3 & 51.1 & 1.0 \\
Monounsaturated & 22.5 & 27.6 & 0.8 \\
n6-PUFA & 13.8 & 15.3 & 0.9 \\
n3-PUFA & 10.5 & 4.5 & 2.3 \\
Total & 100 & 100 & \\
n3-LCP & 11.9 & 6.30 & 1.9 \\
C20:5 n-3 & 0.54 & 0.14 & 4.0 \\
C22:5 n-3 & 0.25 & 0.12 & 1.9 \\
C22:6 n-3 & 9.58 & 4.09 & 2.3 \\
n6-LCP & 13.2 & 14.46 & 0.9 \\
C20:4 n-6 & 8.25 & 9.13 & 0.9 \\
C22:5 n-6 & 1.44 & 1.86 & 0.9 \\
\hline
\end{tabular}

PUFA: polyunsaturated fatty acid; LCP: long-chain polyunsaturated fatty acid ( $>18$ carbons in length).

neuroprotectin D1) (21). Neuroprotectin D1 has also been shown to down-regulate proinflammatory gene expression (21) and up-regulate antiapoptotic protein expression (22). DHA supplementation of human foetal retinal explants incubated for 14 days in bovine serum modified the fatty acid composition in a similar manner to that observed during dietary deficiency. A major drop in DHA content and a rise in 22:5 n-6, the correspondingly most unsaturated fatty acid derived from linoleic acid, were noted (Table 1). The modification of fatty acid composition was accompanied by changes in the pattern of gene expression in human retinal explants compatible with anti-inflammatory effects. Compared with oleic acid, DHA had a marked effect on the expression of genes related to eicosanoid production and in early events of the inflammatory response. DHA exposure resulted in increased expression of the genes for lipocortin, which inhibits arachidonic acid release, and phospholipid hydroperoxide glutathione peroxidase, a negative regulator of lipoxygenase activity. The expression of the gene for leukotriene $\mathrm{A}_{4}$ hydrolase, an enzyme that catalyses leukotriene $\mathrm{B}_{4}$ synthesis, was markedly reduced. In contrast, oleic acid had no significant effects on the expression pattern of these genes (23) (Fig. 1).

Finally, in a mouse model of Alzheimer's disease (Tg2576), dietary DHA deficiency was shown to induce a decline in DHA content of the frontal cortex which was associated with an increase in the fractin/actin ratio and a decreased content of key proteins related to postsynaptic processing. Supplementation of these adult mice with DHA increased DHA brain content, protected them in part from the adverse cellular/biochemical effects and resulted in improved cognitive performance (24).

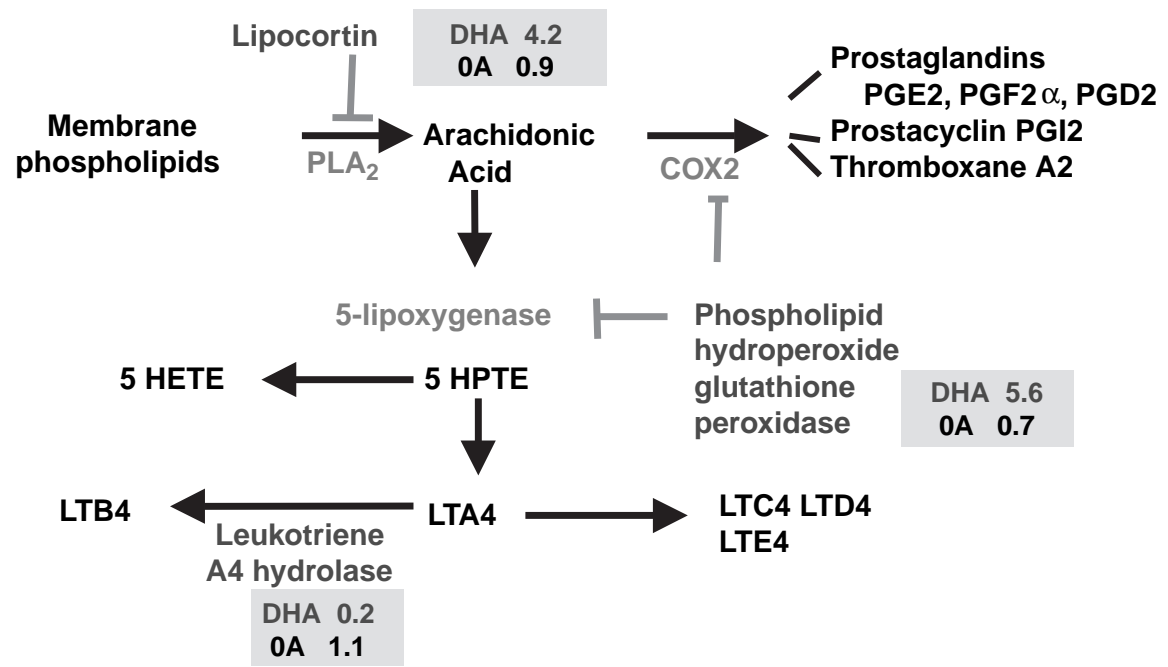

Fig. 1. Effect of docosahexaenoic acid (DHA) and oleic acid (OA) supplementation on the expression of genes involved in eicosanoid production and in early events of the inflammatory response. Numbers indicate the ratio of the corresponding mRNA in retina explants exposed to DHA or OA compared with non-supplemented. DHA exposure resulted in increased expression of the genes for lipocortin and phospholipid hydroperoxide glutathione peroxidase. The expression of the gene for leukotriene $\mathrm{A}_{4}$ hydrolase was markedly reduced by DHA. OA induced minor non-significant changes. PLA 2 : phospholipase $\mathrm{A}_{2}$; COX2: cyclooxygenase-2; HPTE:; HETE: hydroxyeicosatetraenoic acid; LT: leukotriene; PG: prostaglandin. (Based on data published in ref. 23.) 
While epidemiological and mechanistic evidence supporting a role for n-3 LCPs in the maintenance of cognitive health in older age is encouraging, there are currently no data from randomized trials on which firm dietary recommendations can be based (25). The authors are currently conducting a large randomized controlled trial in a group of cognitively normal people aged 70-79 years at baseline, to assess whether an n-3 LCP supplement will preserve retinal function and prevent the cognitive decline observed during ageing (26).

\section{Conclusions}

Despite the impressive documentation of DHArelated health benefits, the cellular and molecular mechanisms for its action are still insufficiently understood. The evidence reviewed in this paper indicates that the effects of DHA on neurodevelopment are mediated not only by their known effect on membrane biophysical properties and the corresponding electrophysiological correlates, but also by effects on neural cell growth, differentiation and functional maturation, and by modulating gene expression in the developing retina and brain. In the ageing brain, n-3 LCPs appear crucial in ensuring optimal eicosanoid balance and potentially in their recently discovered neuroprotective actions. In conclusion, LCPs are conditionally essential nutrients to ensure adequate neurodevelopment and optimal brain ageing in humans.

\section{References}

1. Uauy R, Hoffman DR, Peirano P, Birch DG, Birch EE. Essential fatty acids in visual and brain development. Lipids 2001; 36: 885-95.

2. Uauy R, Dangour AD. Nutrition in brain development and ageing: the role of essential fatty acids. Nutr Rev 2006; 64: S24-33.

3. Litman BJ, Mitchell DC. A role for phospholipid polyunsaturation in modulating membrane protein function. Lipids 1996; 31: S193-7.

4. Calder PC. n-3 Polyunsaturated fatty acids, inflammation, and inflammatory diseases. Am J Clin Nutr 2006; 83: S1505-19.

5. Oppenheim RW. Cell death during development of the nervous system. Annu Rev Neurosci 1991; 14: 453-501.

6. Rotstein N, Aveldaño MI, Barrantes FJ, Roccamo AM, Politi LE. Apoptosis of retinal photoreceptors during development in vitro: protective effect of docosahexaenoic acid. J Neurochem 1997; 69: 504-13.

7. Rotstein N, Aveldaño MI, Barrantes FJ, Politi LE. Docosahexaenoic acid is required for the survival of rat retinal photoreceptors in vitro. J Neurochem 1996; 66: $1851-9$.

8. Kishida E, Yano M, Kasahara M, Masuzawa Y. Distinctive inhibitory activity of docosahexaenoic acid against sphingosine-induced apoptosis. Biochim Biophys Acta 1998; 1391: 401-8.

9. Kim H-Y, Akbar M, Lau A, Edsall L. Inhibition of neuronal apoptosis by docosahexanoic acid (22:6n-3). J Biol Chem 2000; 275: 35215-23.

10. Galli C, Trzeciak HI, Paoletti R. Effects of dietary fatty acids on the fatty acid composition of brain ethanolamine phosphoglyceride: reciprocal replacement of $n-6$ and n-3 polyunsaturated fatty acids. Biochim Biophys Acta 1971; 248: 449-54.

11. Greiner RC, Winter J, Nathanielsz PW, Brenna JT. Brain docosahexaenoate accretion in fetal baboons: bioequivalence of dietary alpha-linolenic and docosahexaenoic acids. Pediatr Res 1997; 42: 826-34.

12. Llanos A, Li Y, Mena P, Salem N Jr, Uauy R. Infants with intrauterine growth restriction have impaired formation of docosahexaenoic acid in early neonatal life: a stable isotope study. Pediatr Res 2005; 58: 735-40.

13. Soderberg M, Edlund C, Kristensson K, Dallner G. Lipid compositions of different regions of the human brain during aging. J Neurochem 1990; 54: 415-23.

14. Suzuki H, Park SJ, Tamura M, Ando S. Effect of the long-term feeding of dietary lipids on the learning ability, fatty acid composition of brain stem phospholipids and synaptic membrane fluidity in adult mice: a comparison of sardine oil diet with palm oil diet. Mech Aging Dev 1998; 101: 119-28.

15. Kalmijn S, van Boxtel MP, Ocke M, Verschuren WM, Kromhout D, Launer LJ. Dietary intake of fatty acids and fish in relation to cognitive performance at middle age. Neurology 2004; 62: 275-80.

16. Kalmijn S, Feskens EJ, Launer LJ, Kromhout D. Polyunsaturated fatty acids, antioxidants, and cognitive function in very old men. Am J Epidemiol 1997; 145: 33-41.

17. Barberger-Gateau P, Letenneur L, Deschamps V, Peres K, Dartigues JF, Renaud S. Fish, meat, and risk of dementia: cohort study. BMJ 2002; 325: 932-3.

18. Uauy R, Valenzuela A. Marine oils: the health benefits of n-3 fatty acids. Nutrition 2000; 16: 680-4.

19. O’Brien JT, Erkinjuntti T, Reisberg B, Roman G, Sawada T, Pantoni L, et al. Vascular cognitive impairment. Lancet Neurology 2003; 2: 89-98.

20. Vermeer SE, Prins ND, den Heijer T, Hofman A, Koudstaal PJ, Breteler MM. Silent brain infarcts and the risk of dementia and cognitive decline. $\mathrm{N}$ Engl $\mathrm{J}$ Med 2003; 348: 1215-22.

21. Marcheselli VL, Hong S, Lukiw WJ, Tian XH, Gronert $\mathrm{K}$, Musto A, et al. Novel docosanoids inhibit brain ischemia-reperfusion-mediated leukocyte infiltration and pro-inflammatory gene expression. J Biol Chem 2003; 278: 43807-17.

22. Mukherjee PK, Marcheselli VL, Serhan CN, Bazan NG. Neuroprotectin D1: a docosahexaenoic acid derived docosatriene protects human retinal pigment epithelial 
cells from oxidative stress. Proc Natl Acad Sci USA 2004; 101: 8491-6.

23. Rojas CV, Martinez JI, Flores I, Hoffman DR, Uauy R. Gene expression analysis in human fetal retinal explants treated with docosahexaenoic acid. Invest Ophthalmol Vis Sci 2003; 44: 3170-7.

24. Calon F, Lim GP, Yang F, Morihara T, Teter B, Ubeda $\mathrm{O}$, et al. Docosahexaenoic acid protects from dendritic pathology in an Alzheimer's disease mouse model. Neuron 2004; 43: 633-45.

25. Lim WS, Gammack JK, Van Niekerk J, Dangour AD. Omega 3 fatty acid for the prevention of dementia. Cochrane Database Syst Rev 2006; Issue 1. Article No. CD005379.pub2. DOI:10.1002/14651858.CD0053 79.pub2.

26. Dangour AD, Clemens F, Elbourne D, Fasey N, Fletcher AE, Hardy $\mathrm{P}$, et al. A randomised controlled trial investigating the effect of $n-3$ long-chain polyunsaturated fatty acid supplementation on cognitive and retinal function in cognitively healthy older people: the Older People And n-3 Long-chain polyunsaturated fatty acids (OPAL) study protocol [ISRCTN72331636]. Nutrition Journal 2006; 5: 20.

\section{Ricardo Uauy}

Nutrition and Public Health Intervention Research Unit London School of Hygiene \& Tropical Medicine

Keppel Street

London WCIE 7HT

UK

E-mail: ricardo.uauy@lshtm.ac.uk 